\title{
Programmed death-ligand 1 expression is associated with fibrosarcomatous transformation of dermatofibrosarcoma protuberans
}

\author{
KENJI TSUCHIHASHI ${ }^{1}$, HITOSHI KUSABA ${ }^{1}$, YUICHI YAMADA ${ }^{2}$, YUTA OKUMURA ${ }^{1}$, HOZUMI SHIMOKAWA ${ }^{3}$, \\ MASATO KOMODA ${ }^{4}$, KEITA UCHINO ${ }^{3}$, TOMOYASU YOSHIHIRO ${ }^{1}$, NOBUHIRO TSURUTA ${ }^{1}$, \\ FUMIYASU HANAMURA $^{1}$, KYOKO INADOMI ${ }^{1}$, MAMORU ITO ${ }^{1}$, KOSUKE SAGARA $^{1}$, MICHITAKA NAKANO ${ }^{1}$, \\ KENTA NIO $^{1}$, SHUJI ARITA ${ }^{5}$, HIROSHI ARIYAMA ${ }^{1}$, KENICHI KOHASHI ${ }^{2}$,

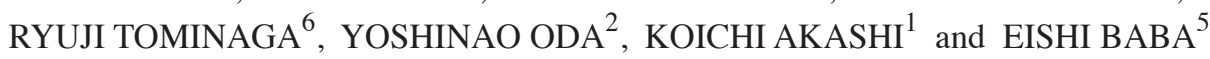

Departments of ${ }^{1}$ Medicine and Biosystemic Sciences and ${ }^{2}$ Anatomic Pathology and Pathological Sciences, Graduate School of Medical Sciences, Kyushu University, Fukuoka 812-8582; ${ }^{3}$ Department of Oncology, Kyushu National Medical Center, Fukuoka 810-8563; ${ }^{4}$ Department of Gastrointestinal and Medical Oncology, Kyushu Hospital Organization Kyushu Cancer Center, Fukuoka 811-1395; ${ }^{5}$ Department of Comprehensive Clinical Oncology, Faculty of Medical Sciences, Kyushu University, Fukuoka 812-8582; ${ }^{6}$ Department of Cardiovascular Surgery, Fukuoka Wajiro Hospital, Fukuoka 811-0213, Japan

Received January 20, 2017; Accepted March 10, 2017

DOI: $10.3892 / \mathrm{mco} .2017 .1197$

\begin{abstract}
Dermatofibrosarcoma protuberans (DFSP) is a locally invading tumor, characterized by the presence of the collagen type I $\alpha 1(C O L 1 A 1)$-platelet-derived growth factor $(P D G F) \beta$ fusion gene. We herein report the case of a 31-year-old man with a history of resection of an abdominal wall DFSP. The patient presented with chest pain and a computed tomography scan revealed a large mass in the posterior mediastinum and another mass in the right lung. The mediastinal mass was a sarcomatous lesion expressing the COL1A1-PDGF $\beta$ fusion gene, suggesting that it represented a metastasis of the DFSP following fibrosarcomatous (FS) transformation. Following resection of the mediastinal metastasis and subsequent radiotherapy, the mass in the right lung was also resected. Due to the emergence of pleural and pancreatic tail metastases, the patient was treated with a combination therapy of adriamycin and ifosfamide. After five courses, the disease progressed and the patient was subsequently treated with pazopanib for $\sim 2$ months until further progression. Three years after the diagnosis of the mediastinal metastasis of DFSP, the patient was referred to another hospital for palliative care. The expression of programmed cell death 1 ligand (PD-L1) in the primary and metastatic tumors was investigated: PD-L1
\end{abstract}

Correspondence to: Professor Eishi Baba, Department of Comprehensive Clinical Oncology, Faculty of Medical Sciences, Kyushu University, 3-1-1 Maidashi, Higashi-ku, Fukuoka 812-8582, Japan

E-mail: e-baba@c-oncology.med.kyushu-u.ac.jp

Key words: dermatofibrosarcoma protuberans, sarcomatous transformation, programmed death-ligand 1 , metastasis expression was detected in the metastasis but not in the primary tumor. Given that the metastatic tumor exhibited FS transformation (DFSP-FS), PD-L1 expression may be induced by FS transformation, contributing to the metastasis through escape from immune surveillance. Further investigation of the PD-L1 pathway in DFSP and DFSP-FS in primary as well as metastatic sites is required to evaluate the clinical efficacy of therapies targeting the PD-L1 signaling cascade.

\section{Introduction}

The relatively rare dermatofibrosarcoma protuberans (DFSP) is an invasive but slow-growing neoplasm of the dermis. Whereas local recurrence is frequently observed, metastasis is uncommon (1). DFSP is characterized by the rearrangement of chromosomes 17 and 22, leading to fusion of the genes encoding for collagen type I $\alpha 1$ (COL1A1) and plateletderived growth factor $\beta$ (PDGF $\beta)$ The expression of the COL1A1-PDGF $\beta$ fusion gene results in increased secretion of PDGF-BB, which activates the autocrine signaling of the PDGF receptor $\beta$ (PDGFR $\beta$ ) (2). DFSP occasionally exhibits fibrosarcomatous (FS) transformation, resulting in a more aggressive phenotype, which is associated with a risk of metastasis $>10$-fold that of DFSP $(1,3)$. The precise molecular mechanism underlying the transformation of DSFP to DSFP-FS has not been fully elucidated $(4,5)$.

The signaling pathway initiated by programmed death-ligand 1 (PD-L1, CD274) and its receptor programmed death 1 (PD-1, CD279) regulates critical interactions between tumor cells and the host immune system, which fosters tumor immune escape (6). Therefore, targeted therapies against the PD-1/PD-L1 pathway have been proposed in various neoplasms. However, the PD-1/PD-L1 pathway in DFSP has not been yet characterized and altered expression of PD-L1 in 

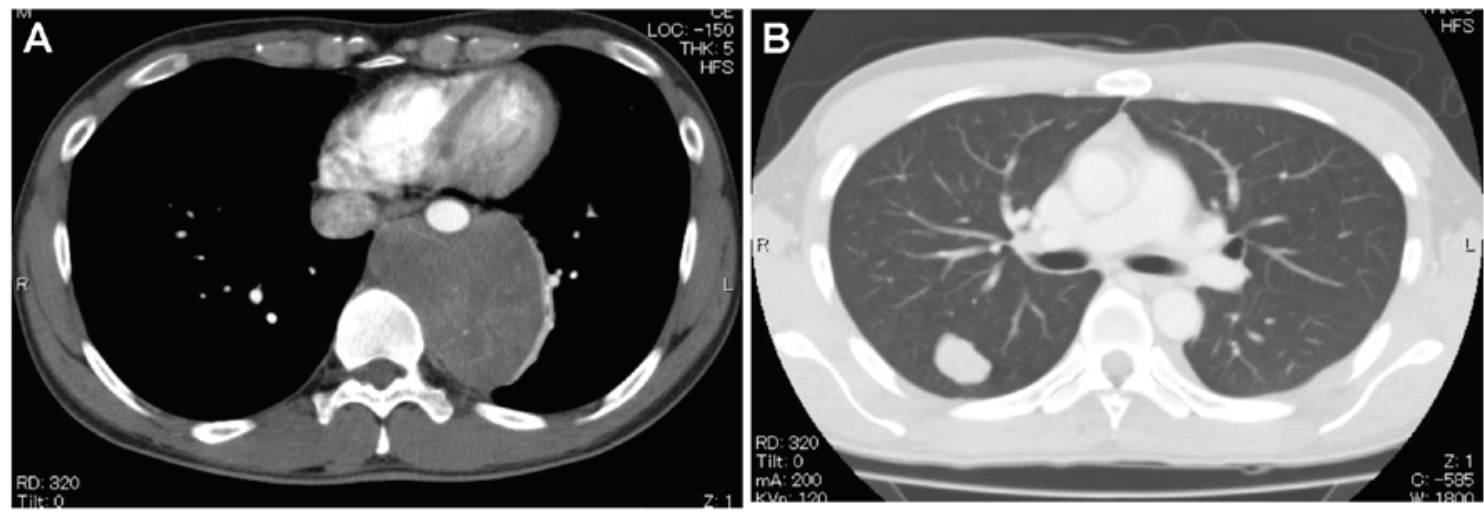

Figure 1. Computed tomography scan showing (A) the posterior mediastinal tumor and (B) the tumor in the right lung.
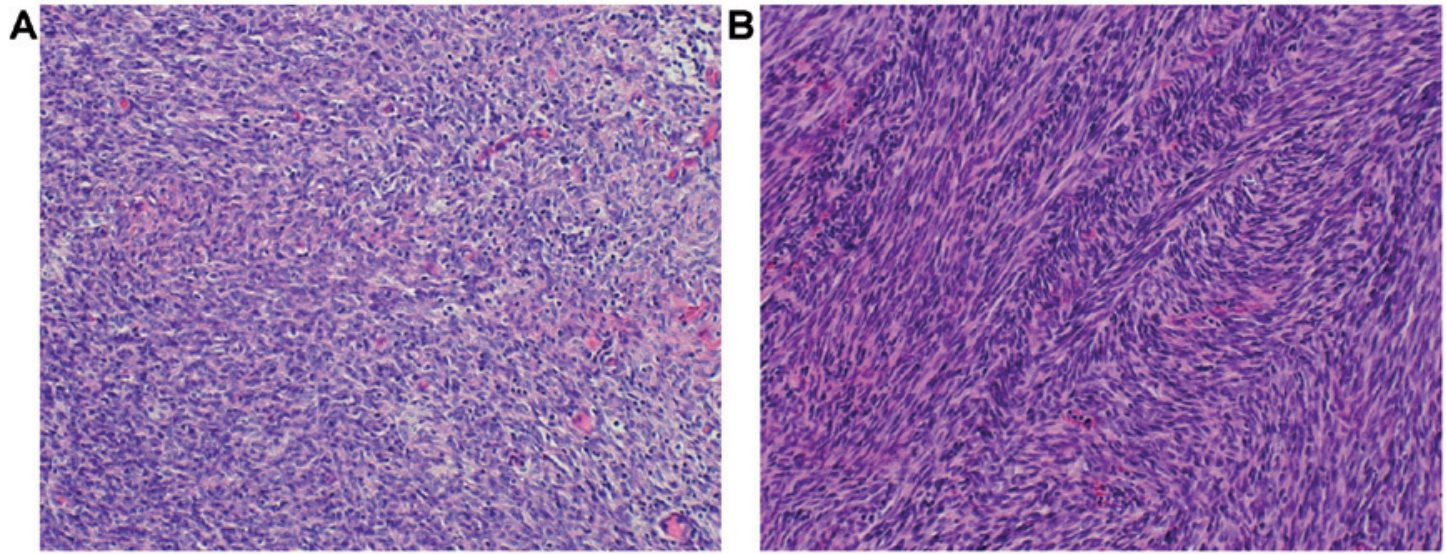

Figure 2. Hematoxylin and eosin staining of (A) the primary tumor and (B) the posterior mediastinal metastasis (magnification, x200).

DFSP-FS has not been reported. In the present study, the presence of the COL1A1-PDGF $\beta$ fusion gene was confirmed in DFSP metastases and the expression of PD-L1 was compared between the primary DFSP site and the DFSP-FS metastasis.

\section{Case report}

A 31-year-old man first presented at another hospital with chest pain in March, 2010 The patient had previously undergone surgical resection of DFSP in the abdominal wall 4 years prior. The tumor was completely resected and no adjuvant therapy was performed. A computed tomography (CT) scan revealed a mass sized $80 \times 60 \times 80 \mathrm{~mm}$ in the posterior mediastinum in contact with the aortic wall, and a second mass sized 28x18x18 mm in the right lung (Fig. 1). The patient was then referred to the Kyushu University Hospital (Fukuoka, Japan) in March, 2010. Endoscopy-guided biopsy of the mass in the posterior mediastinum through the esophagus revealed a proliferation of atypical spindle-shaped cells, suggesting DFSP metastasis. In June 2010 surgical resection of the mass of the posterior mediastinum and replacement of the descending aorta were performed, followed by adjuvant radiation therapy at a dose of $30 \mathrm{~Gy}$. Pathological examination of the resected tumor revealed a herringbone-like growth pattern, indicating FS transformation of DFSP, as it was not detected in the primary tumor (Fig. 2). Furthermore, the tumor was immunohistochemically positive for CD34 and PDGFR $\beta$, but negative for epithelial membrane antigen, cytokeratin (CK)7, CK19, $\alpha$-smooth muscle actin, S-100 and c-kit. Reverse transcription polymerase chain reaction analysis revealed the presence of the COL1A1 (exon47)-PDGF $\beta$ fusion gene, compatible with the diagnosis of metastatic DFSP. Karyotype analysis by G-banding revealed the presence of $t(17 ; 22)$ in two different sites in the resected tumor. In October 2010, thoracoscopic partial resection of the right upper lobe of the lung was performed. However, 10 months after the operation, small nodules were detected in the right pleura, at the level of the 7th thoracic vertebra, and in the tail of the pancreas. Four months after the appearance of the tumors, chemotherapy with adriamycin and ifosfamide (AI) was initiated, as these tumors enlarged. After five courses of AI therapy, the disease progressed. The best overall response was stable disease. Treatment with pazopanib, a multi-target tyrosine kinase inhibitor of vascular endothelial growth factor receptor, PDGFR, fibroblast growth factor receptor and c-Kit was initiated and continued for 4 months until disease progression. Apart from grade 1 diarrhea according to the Common Terminology Criteria for Adverse Events v4.0 (https://www.eortc.be/services/doc/ctc/ CTCAE_4.03_2010-06-14_QuickReference_5x7.pdf), adverse events were not observed. The patient was referred to a specialized hospital for palliative care in May, 2013.

The expression of PD-L1 was immunohistochemically assessed in the primary tumor of the abdominal wall and in the mediastinal metastatic site using three anti-PD-L1 
A
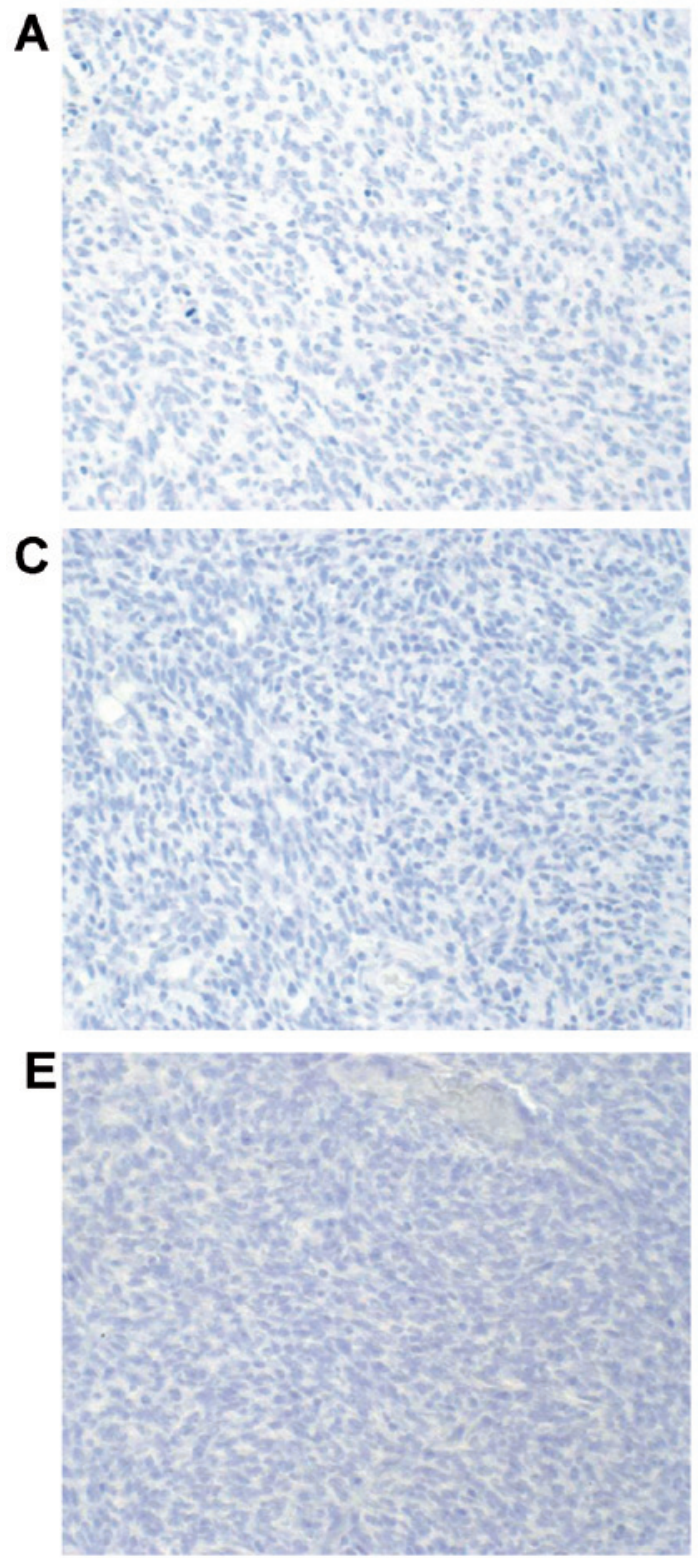

B

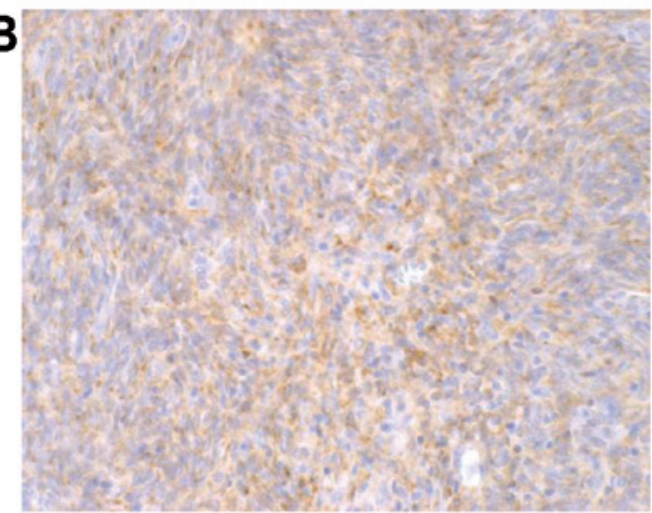

D

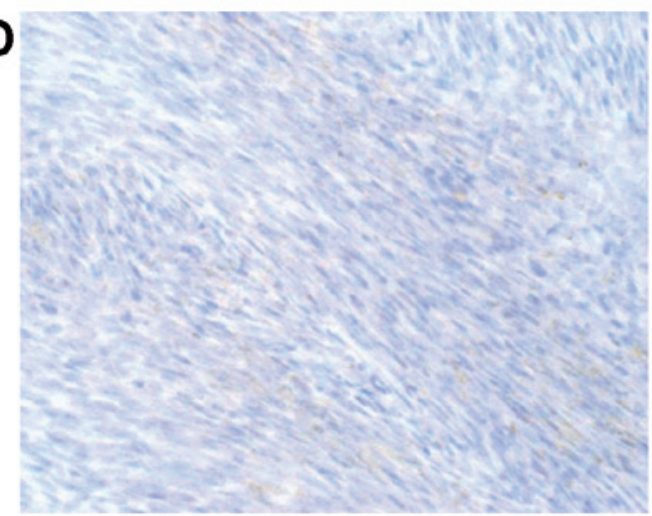

$\mathbf{F}$

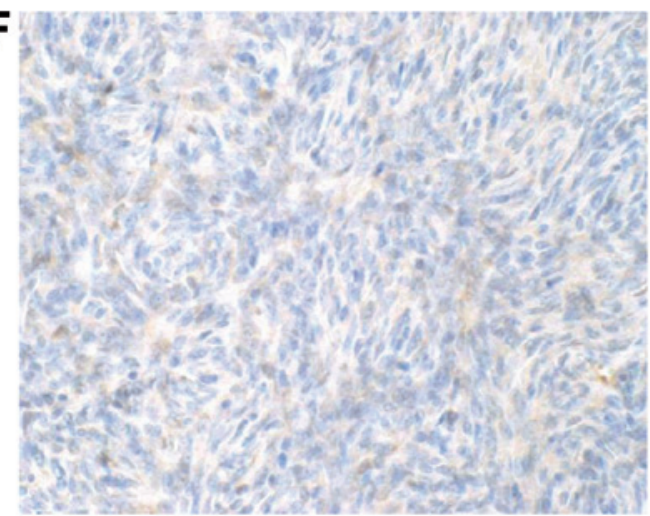

Figure 3. Programmed death-ligand 1 expression analysis in the primary and metastatic sites using (A and B) clone E1L3N (Cell Signaling Technology), (C and D) clone SP142 (Spring Bioscience) and (E and F) clone 28-8 (Abcam) antibodies, respectively.

monoclonal antibodies (rabbit monoclonal, clone E1L3N, dilution 1/200, Cell Signaling Technology, Danvers, MA, USA; rabbit monoclonal, clone SP142, dilution 1/200, Spring Bioscience, Pleasanton, CA, USA; and rabbit monoclonal, clone 28-8, dilution 1/100, Abcam, Tokyo, Japan). While there was no staining of the primary tumor, PD-L1 was detected by all antibodies in the metastatic mass (Fig. 3), suggesting that the expression of PD-L1 may be associated with DFSP-FS and maybe involved in the development of metastasis.

\section{Discussion}

DFSP is a slow-growing, invasive tumor, with a high rate of local recurrence; however, distant metastasis is rare (1). In 9-20\% of the cases, DFSP exhibits FS transformation (DFSP-FS), and $14.4-18 \%$ of DFSP-FS harbor metastases $(1,3)$. FS transformation is pathologically characterized by a fascicular pattern on histological examination $(1,3)$.
The main target organs for DFSP-FS metastasis are the lungs, bones, soft tissue and brain $(7,8)$. The pancreas is reported to be a rare metastatic site (9). Our patient had metastases in the posterior mediastinum, lung, pancreas and soft tissue. As posterior mediastinal DFSP metastasis has not been previously reported and the mediastinal tumor in the present case was large, it initially had to be distinguished from primary mediastinal tumors, such as neurogenic tumors and lymphomas. An endoscopic transesophageal biopsy revealed that the mediastinal mass displayed the histological characteristics of fibrosarcoma, supporting the diagnosis of a DFSP metastasis. This was further confirmed by detection of the rearrangement of $\mathrm{t}(17 ; 22)$ and COL1A1-PDGF $\beta$ fusion gene at the molecular level.

The expression of COL1A1-PDGF $\beta$ leads to increased PDGF $\beta$ secretion and, in turn, to the activation of autocrine PDGFR $\beta$ signaling (2), the inhibition of which is effective in suppressing DFSP. Imatinib mesylate inhibits the BCR-ABL 
fusion protein and it also negatively regulates the kinase activity of PDGR $\beta$. Imatinib has been reported to achieve a favorable response rate in DFSP (10), but has not been approved for the treatment of soft tissue sarcomas, including DFSP, in Japan. Pazopanib, a kinase inhibitor against multiple targets, including PDGFR $\beta$ is approved for the treatment of soft tissue sarcomas, although its efficacy as treatment for DFSP remains unknown (11). In the present case, the patient received AI therapy, generally employed for soft tissue sarcomas, and achieved 5 months of progression-free survival. Subsequent treatment with pazopanib was not effective. The possible reasons for the low effectiveness of pazopanib treatment may be that pazopanib does not have a high affinity for PDGFR (12), and/or that FS transformation of DFSP may decrease its sensitivity to chemotherapy $(5,13)$.

Recently, immunotherapy targeting the PD-1/PD-L1 pathway has been reported to be effective against various malignancies (6). PD-1 is a physiological co-inhibitory receptor expressed in T cells, B cells, monocytes and natural killer $\mathrm{T}$ cells. Its ligand, PD-L1, is expressed in antigen-presenting cells, as well as in endothelial cells (14). The interaction between PD-1 and PD-L1 suppresses aberrant autoimmune responses. In turn, the expression of PD-L1 in tumor cells results in their evasion from immune surveillance and tumor progression. Hence, interference with PD-1/PD-L1 signaling activates the immune response against tumors and, in turn, suppresses their progression. In our case, the primary site did not express PD-L1, while the metastatic site did, suggesting that PD-L1 expression may be involved in the development of metastasis through immune escape. Previous studies reported that PD-L1 expression is promoted by various mechanisms, such as activation of the mitogen-activated protein kinase or phosphoinositide 3 kinase/AKT pathway, or epigenetic regulation $(15,16)$. Although the mechanism of FS transformation of DFSP is under investigation and has not been fully elucidated $(4,5,17)$, PD-L1 expression and FS transformation may be induced by the activation of a common signaling pathway or epigenetic changes. Callea et al reported differential PD-L1 expression between primary and metastatic sites in $20 \%$ of renal cell carcinoma cases, suggesting the importance of the assessment of the primary as well as the metastatic sites (18). While it remains unclear whether anti-PD-1/PD-L1 therapy would be effective against DFSP and whether the expression level of PD-L1 is correlated with the efficacy of this treatment, our data indicate that anti-PD1/PD-L1 therapy may suppress the formation or growth of metastasis through upregulation of the immune response.

In summary, we herein reported a case of DFSP with metastasis composed of DFSP-FS. PD-L1 expression was differentially expressed in the primary and metastatic tumors, suggesting that it may contribute to the development of metastases.

\section{Acknowledgements}

The present study was supported by Grant-in-Aid for Young Scientists (B) and the Fukuoka Foundation for Sound Health Cancer Research Fund (K. Tsuchihashi).

\section{References}

1. Hoesly PM, Lowe GC, Lohse CM, Brewer JD and Lehman JS: Prognostic impact of fibrosarcomatous transformation in dermatofibrosarcoma protuberans: A cohort study. J Am Acad Dermatol 72: 419-425, 2015.

2. Shimizu A, O’Brien KP, Sjöblom T, Pietras K, Buchdunger E, Collins VP, Heldin CH, Dumanski JP and Ostman A: The dermatofibrosarcoma protuberans-associated collagen type Ialpha1/platelet-derived growth factor (PDGF) B-chain fusion gene generates a transforming protein that is processed to functional PDGF-BB. Cancer Res 59: 3719-3723, 1999.

3. Liang CA, Jambusaria-Pahlajani A, Karia PS, Elenitsas R, Zhang PD and Schmults CD: A systematic review of outcome data for dermatofibrosarcoma protuberans with and without fibrosarcomatous change. J Am Acad Dermatol 71: 781-786, 2014.

4. Llombart B, Monteagudo C, Sanmartín O, López-Guerrero JA Serra-Guillén C, Poveda A, Jorda E, Fernandez-Serra A, Pellín A, Guillén $\mathrm{C}$, et al: Dermatofibrosarcoma protuberans: A clinicopathological, immunohistochemical, genetic (COL1A1-PDGFB), and therapeutic study of low-grade versus high-grade (fibrosarcomatous) tumors. J Am Acad Dermatol 65: 564-575, 2011.

5. Stacchiotti S, Astolfi A, Gronchi A, Fontana A, Pantaleo MA, Negri T, Brenca M, Tazzari M, Urbini M, Indio V, et al: Evolution of Dermatofibrosarcoma protuberans (DFSP) to DFSP-derived fibrosarcoma (FS-DFSP): An event marked by epithelial mesenchymal transition-like process and 22q loss. Mol Cancer Res 14: 820-829, 2016.

6. Ohaegbulam KC, Assal A, Lazar-Molnar E, Yao Y and Zang X: Human cancer immunotherapy with antibodies to the PD-1 and PD-L1 pathway. Trends Mol Med 21: 24-33, 2015.

7. Rutgers EJ, Kroon BB, Albus-Lutter CE and Gortzak E: Dermatofibrosarcoma protuberans: Treatment and prognosis. Eur J Surg Oncol 18: 241-248, 1992.

8. Cai H, Wang Y, Wu J and Shi Y: Dermatofibrosarcoma protuberans: Clinical diagnoses and treatment results of 260 cases in China. J Surg Oncol 105: 142-148, 2012.

9. Yokoyama Y, Murakami Y, Sasaki M, Morifuji M, Hayashidani Y, Kobayashi T, Sudo T and Sueda T: Pancreatic metastasis of dermatofibrosarcoma protuberans. J Gastroenterol 39: 798-800, 2004.

10. Rubin BP, Schuetze SM, Eary JF, Norwood TH, Mirza S, Conrad EU and Bruckner JD: Molecular targeting of plateletderived growth factor B by imatinib mesylate in a patient with metastatic dermatofibrosarcoma protuberans. J Clin Oncol 20: 3586-3591, 2002.

11. Malhotra B and Schuetze SM: Dermatofibrosarcoma protruberans treatment with platelet-derived growth factor receptor inhibitor: A review of clinical trial results. Curr Opin Oncol 24: 419-424, 2012.

12. Hamberg P, Verweij J and Sleijfer S: (Pre-)clinical pharmacology and activity of pazopanib, a novel multikinase angiogenesis inhibitor. Oncologist 15: 539-547, 2010.

13. Tazzari M, Indio V, Vergani B, De Cecco L, Rini F, Negri T, Camisaschi C, Fiore M, Stacchiotti S, Dagrada GP, et al: Adaptive Immunity in Fibrosarcomatous Dermatofibrosarcoma Protuberans and Response to Imatinib Treatment. J Invest Dermatol 8: 328, 2016.

14. Zou W, Wolchok JD, Chen L: PD-L1 (B7-H1) and PD-1 pathway blockade for cancer therapy: Mechanisms, response biomarkers, and combinations. Sci Transl Med 8:328rev, 2016.

15. Chen J, Jiang CC, Jin L and Zhang XD: Regulation of PD-L1: A novel role of pro-survival signalling in cancer. Ann Oncol 27: 409-416, 2016

16. Cortez MA, Ivan C, Valdecanas D, Wang X, Peltier HJ, Ye Y, Araujo L, Carbone DP, Shilo K, Giri DK, et al: PDL1 Regulation by p53 via miR-34. J Natl Cancer Inst 108: djv303, 2015.

17. Takahira T, Oda Y, Tamiya S, Yamamoto H, Kawaguchi K, Kobayashi C, Oda S, Iwamoto Y and Tsuneyoshi M: Microsatellite instability and p53 mutation associated with tumor progression in dermatofibrosarcoma protuberans. Hum Pathol 35: 240-245, 2004.

18. Callea M, Albiges L, Gupta M, Cheng SC, Genega EM, Fay AP, Song J, Carvo I, Bhatt RS, Atkins MB, et al: Differential Expression of PD-L1 between Primary and Metastatic Sites in Clear-Cell Renal Cell Carcinoma. Cancer Immunol Res 3: 1158-1164, 2015. 